\title{
BMI1 Inhibitors Down-regulate NOTCH Signaling and Suppress Proliferation of Acute Leukemia Cells
}

\author{
MIKA OHTAKA, MAI ITOH and SHUJI TOHDA \\ Department of Laboratory Medicine, Tokyo Medical and Dental University, Tokyo, Japan
}

\begin{abstract}
Background/Aim: B cell-specific Moloney murine leukemia virus integration site 1 (BMII) is up-regulated in several cancers; therefore, we investigated the effects of BMII inhibitors on leukemia cells. Materials and Methods: Four acute myeloid leukemia and two T-lymphoblastic leukemia cell lines were treated with BMII inhibitors artemisinin, PRT4165, and PTC-209 and analyzed for cell proliferation and gene expression by microarray and immunoblotting. Results: PTC-209 and PRT4165 suppressed the growth of all cell lines through apoptosis.Artemisinin acted only on Jurkat cells. BMII inhibitors and BMI1specific siRNA down-regulated the expression of NOTCH signaling proteins NOTCH1, HES1, and MYC. All but one cell lines did not have the cyclin-dependent kinase inhibitor $2 A(C D K N 2 A)$ gene targeted by BMI1, thus the inhibitors acted through CDKN2A-independent pathways. Conclusion: BMII inhibition suppressed proliferation of leukemia cells through NOTCH signaling which functions downstream of BMIl, suggesting that BMII inhibitors can be candidate targeted drugs against leukemia.
\end{abstract}

Polycomb group (PcG) proteins are transcriptional repressors playing key roles in self-renewal of stem cells through chromatin remodelling. PcG proteins function within two multiprotein complexes called polycomb repressive complex (PRC) 1 and PRC2, which regulate different steps of gene expression. PRC2 binds to the target genes and trimethylates histone H3 lysine 27 (H3K27me3) in chromatin (1), whereas $\mathrm{PRC} 1$ recognizes $\mathrm{H} 3 \mathrm{~K} 27 \mathrm{me} 3$, and $\mathrm{E} 3$ ubiquitin ligase RING1A/B within PRC1 ubiquitinates histone H2A at lysine 119 (2). As a result, concerted activity of PRC1 and PRC2 leads to silencing of target genes linked to tumour development (3).

Correspondence to: Prof. Shuji Tohda, Department of Laboratory Medicine, Tokyo Medical and Dental University, Yushima 1-5-45, Bunkyo-Ku, Tokyo 113-8519, Japan. Tel: +81 358035334, Fax: +81 358035629, e-mail: tohda.mlab@tmd.ac.jp

Key Words: BMI1, leukemia, NOTCH, CDKN2A.
B cell-specific Moloney murine leukemia virus integration site 1 (BMI1) is a key regulatory component of PRC1 (4) known to promote cell proliferation by inhibiting expression of various genes such as cyclin-dependent kinase inhibitor 2A $(C D K N 2 A)$ which encodes CDK inhibitor p16 ${ }^{\mathrm{INK} 4 \mathrm{~A}}$ and tumour suppressor p14 ${ }^{\mathrm{ARF}}$. BMI1 was shown to be upregulated in several cancers, including acute leukemia (4-6), and a novel small molecule inhibitor of BMI1, PTC-209, has demonstrated inhibitory activity in human colorectal cancer (7), acute leukemia (6), and myeloma (8).

In this study, we investigated the role of BMI1 in leukemia cell growth using three BMI1 inhibitors, artemisinin, PTC-209, and PRT4165 and six leukemia cell lines, THP-1, TMD7, OCI/AML2, OCI/AML5, Jurkat and KOPT-K1. We also examined the underlying molecular mechanisms, focusing on NOTCH signaling which is crucial for self-renewal of leukemia stem cells (9), and analyzed the expression of NOTCH, cleaved $\mathrm{NOTCH}$, the hairy and enhancer of split 1 (HES1), and v-myc myelocytomatosis viral oncogene homolog (MYC).

\section{Materials and Methods}

Cell lines and BMI1 inhibitors. We used six leukemia cell lines (THP-1, TMD7, OCI/AML772, and OCI/AML5 derived from AML, and Jurkat and KOPT-K1 derived from T-ALL) and normal lymphocytes from two healthy volunteers who provided informed consent. THP-1 was obtained from the Health Science Research Resource Bank (Osaka, Japan), TMD7 was established in our laboratory (10), and OCI/AML2 and OCI/AML5 were established at the Ontario Cancer Institute (11). Jurkat cells were purchased from the European Collection of Cell Cultures (Porton Down, Wiltshire, UK), and KOPT-K1 was donated by Drs. Harashima and Orita, Fujisaki Cell Center (Okayama, Japan). Cells were cultured in RPMI-1640 supplemented with $10 \%$ fetal bovine serum in a humidified 5\%-CO2 atmosphere. BMI1 inhibitors artemisinin, PTC209, and PRT4165 were purchased from Sigma (St. Louis, MO, USA), Xcess Biosciences Inc. (San Diego, CA, USA), and Axon Medchem (Groningen, The Netherlands), respectively, and dissolved in dimethyl sulfoxide (DMSO). The inhibitors were chosen based on previous results showing that artemisinin and PTC-209 suppressed BMI1 mRNA expression $(7,12)$ and PRT4165 inhibited BMI 1 by down-regulating BMI1/RING1A self-ubiquitination $(12,13)$. 


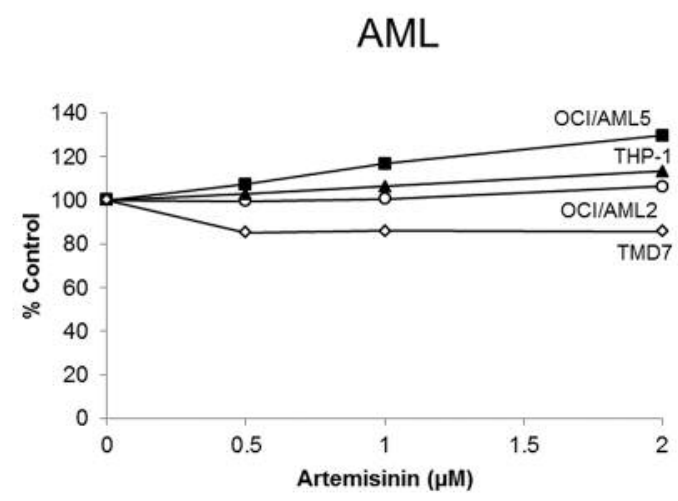

T-ALL Lymphocytes
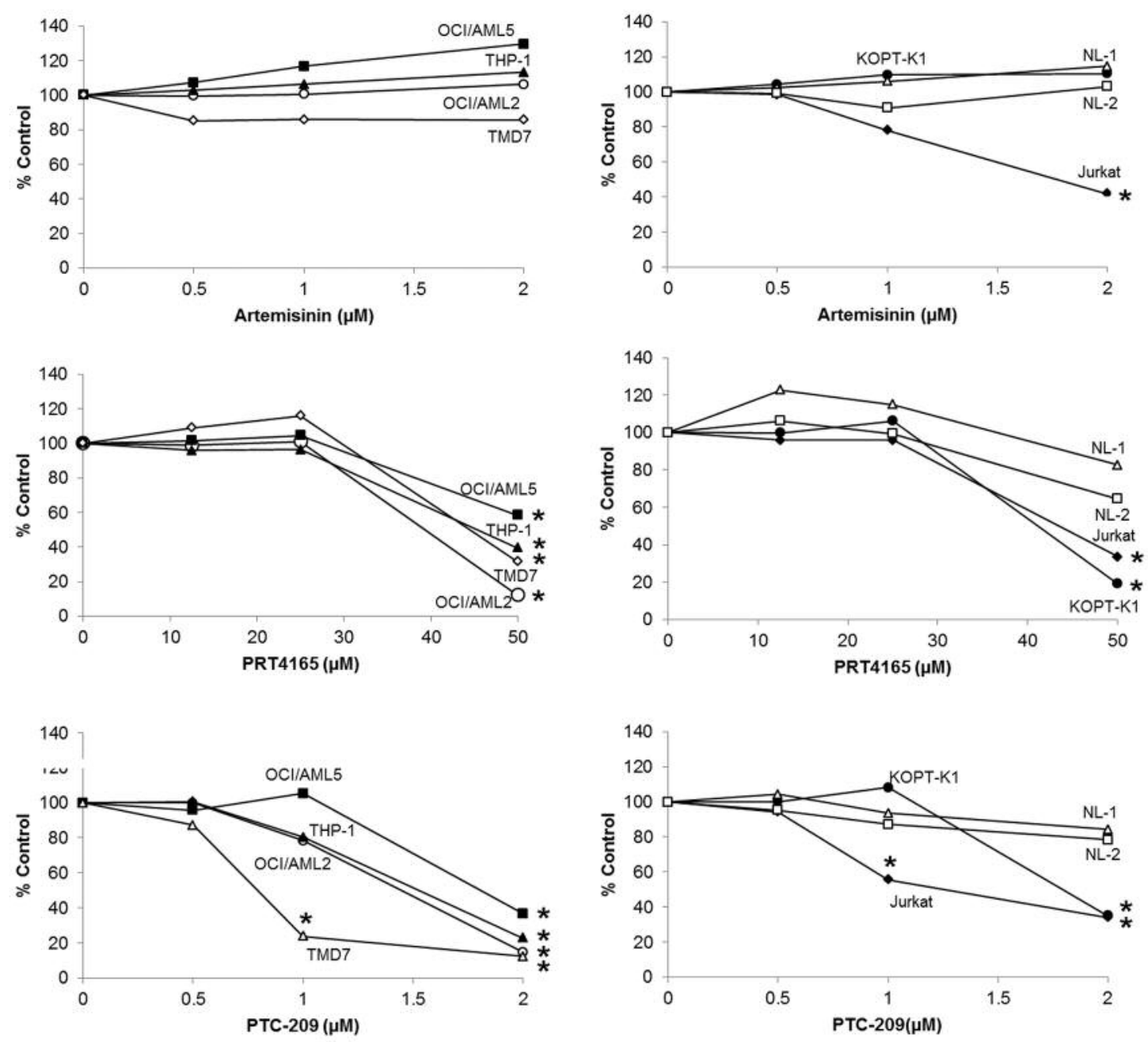

Figure 1. Effects of BMI1 inhibitors on cell growth. Cells were cultured with the indicated concentrations of inhibitors for three days and cell growth was evaluated using a colorimetric assay. The results are expressed as a percentage of the mean $O D$ in inhibitor-treated cells normalized to that in control (DMSO-treated) cells. NL: Normal lymphocytes. * $p<0.05$ compared to control.

Cell growth assay. Cell proliferation was assessed using the colorimetric WST-8 assay (Dojindo Laboratories, Kumamoto, Japan). Cells were cultured with or without increasing concentrations of the inhibitors in 96-well culture plates for three days. Then, WST8 and 1-methoxy-5-methylphenazinium methyl sulphate were added, and optical density (OD) was measured using an enzyme-linked immunosorbent assay plate reader. Relative cell proliferation was calculated as the percentage of the mean OD value normalized to that of the control. The effects of the inhibitors on cell morphology and apoptosis were examined in cytospin preparations stained with Wright's stain and observed under a microscope.

Apoptosis assay and flow cytometry. Cells treated with BMI1 inhibitors were stained with Annexin V-FITC and propidium iodide, and analysed using a FACS Calibur cytometer (BD Biosciences, Franklin Lakes, NJ, USA) to evaluate the induction of apoptosis.

Western blotting. Protein expression was examined by immunoblotting. After culturing with the inhibitors, cells were harvested and lysed. The lysates were subjected to sodium dodecyl sulphate-polyacrylamide gel electrophoresis (SDS-PAGE) and immunoblotted with antibodies against BMI1, NOTCH1, cleaved NOTCH1 (Val1744), HES1, MYC, caspase-3 (Cell Signaling Technology, Danvers, MA, USA), p16 ${ }^{\mathrm{INK}} 4 \mathrm{~A}, \mathrm{p} 14^{\mathrm{ARF}}$ (Santa Cruz Biotechnology, Santa Cruz, CA, USA), and $\alpha$-tubulin (Abcam, Cambridge, MA, USA) which was used as a loading control. Immunoreactive bands were detected using an Pierce Enhanced Chemiluminescent Western Blotting kit (Pierce Biotechnology, 


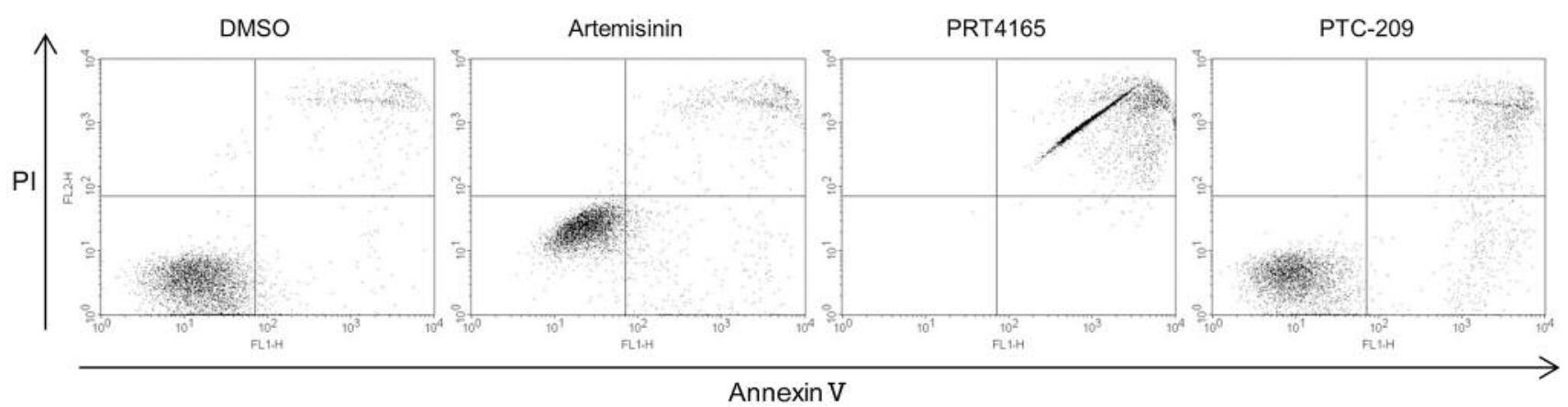

Figure 2. Apoptosis assay of Jurkat cells treated with BMI1 inhibitors. Cells were cultured with $2 \mu M$ artemisinin, 50 $\mu M$ PRT4165, and $2 \mu M$ PTC-209 for 48 h, stained with Annexin V-FITC and propidium iodide (PI), and analysed for apoptosis by flow cytometry.

Rockford, IL, USA). Each assay was repeated more than twice to ascertain reproducibility.

BMI1 knockdown by siRNA. To confirm specificity of the effects exerted by BMI1 inhibitors, we performed BMII knockdown by small interfering RNA (siRNA). Three different pre-designed siRNAs (Stealth siRNA ${ }^{\mathrm{TM}}$ ) targeting BMII (HSS 101038, 101039, and 101040) were purchased from Life Technologies (Carlsbad, CA, USA); stealth RNAi negative control Duplex was used as a control. Cells were transfected with $40 \mathrm{nM}$ of each siRNA using the Neon ${ }^{\mathrm{TM}}$ pipette tip chamber-based electroporation system (Life Technologies, Carlsbad, CA, USA) according to the manufacturer's instructions, and immediately transferred to culture medium.

Microarray analysis. To assess transcriptional effects of BMI1 inhibitors, changes in mRNA expression were examined by microarray analysis. Jurkat and THP-1 cells were treated with the inhibitors, DMSO, BMI 1 siRNA, or control siRNA for $24 \mathrm{~h}$. Total RNA was extracted using a High Pure RNA isolation kit (Roche Diagnostics, Mannheim, Germany) and used to prepare cyanine-3labeled cRNA which was hybridized to a SurePrint G3 Human GE microarray $8 \times 60 \mathrm{~K}$ v3 (Agilent Technologies, Santa Clara, CA, USA). The expression profile was analysed using the Agilent Feature Extraction 11.5.1.1 software.

\section{Results}

Effects of BMII inhibitors on cell growth and apoptosis. Dose-response curves (Figure 1) showed that artemisinin suppressed proliferation of Jurkat cells, but not of the other cell lines; however, PTC-209 and PRT4165 suppressed growth of all six cell lines. At the same time, none of the BMI1 inhibitors affected the viability of normal lymphocytes in the range of concentrations used.

Analysis of cytospin preparations indicated that the BMI1 inhibitors did not alter cell morphology. However, apoptotic cells with nuclear condensation and apoptotic bodies were detected in cell lines susceptible to the inhibitors (data not shown). Consistent with these results, FACS assay revealed that BMI1 inhibitors induced apoptosis, which corresponded to cell growth retardation (representative data are shown for Jurkat cells in Figure 2).

Effects of BMII inhibitors on signaling proteins. Immunoblotting analysis confirmed BMI1 protein expression in all six cell lines (Figure 3). In addition, TMD7 cells also expressed p16 ${ }^{\text {INK4A }}$ (Figure 3) and p14 ${ }^{\mathrm{ARF}}$ (data not shown), which were not detected in the other cell lines. Treatment with the inhibitors affected the expression and activity of signaling proteins belonging to the NOTCH pathway (Figure 3 and Table I).

Artemisinin down-regulated the expression of BMI, NOTCH1, and cleaved NOTCH1 along with the downstream targets of NOTCH, HES1 and MYC, in Jurkat cells.

PRT4165 suppressed the expression of NOTCH1 in KOPTK1, OCI/AML2, and OCI/AML5 cells. PRT4165 also suppressed the expression of cleaved NOTCH1, MYC, and HES1 in KOPT-K1 cells. OCI/AML2 and OCI/AML5 cells did not express cleaved NOTCH1 and HES1. PRT4165 induced cleavage of caspase-3 in five cell lines except Jurkat cells.

PTC-209 reduced the expression of BMI1 as well as NOTCH1 and MYC in all cell lines. PTC-209 induced caspase-3 cleavage in TMD7 and OCI/AML5 cells.

Effects of BMII knockdown by siRNA on protein expression. As HSS 101038 (5'-UCCUCAUCCACAGUUUCCUC ACAUU3') was the most potent among the examined BMII-specific siRNAs, its effects on protein expression are shown in Figure 4. In Jurkat cells, BMII knockdown decreased the expression of NOTCH1, HES1, and MYC, which is consistent with the effects caused by PTC-209, suggesting that the inhibitors downregulated NOTCH signaling proteins through BMI.

Microarray analysis. In microarray gene expression analysis, we focused on NOTCH signaling genes. Table II shows mRNA $\log 2$ ratios between inhibitor-treated and 


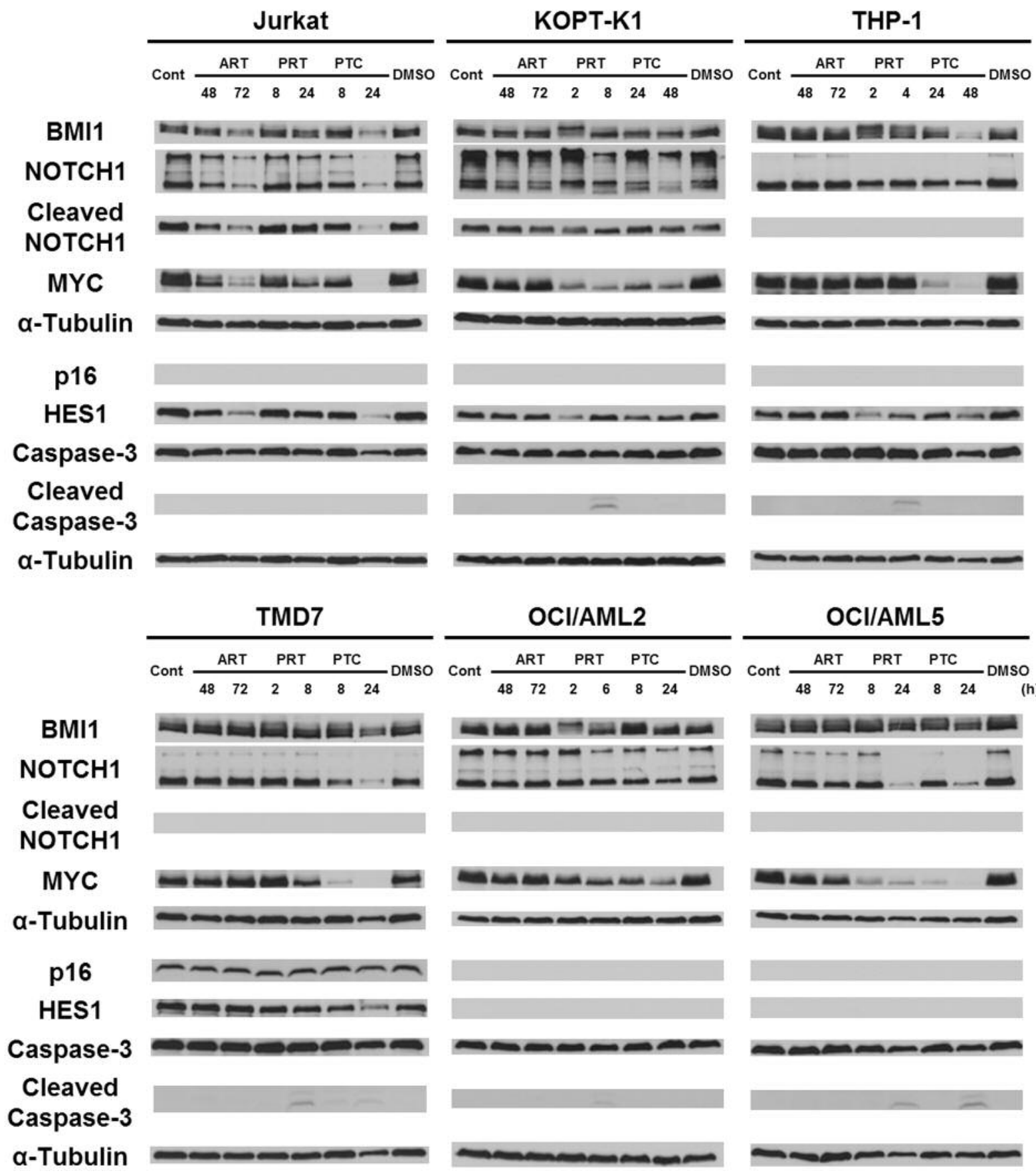

Figure 3. Expression of BMI1, p16INK4A, and NOTCH signaling proteins in leukemia cells treated with BMI1 inhibitors. Cells were cultured with 2 $\mu M$ artemisinin (ART), $50 \mu M$ PRT4165 (PRT), and $2 \mu M$ PTC-209 (PTC) for the indicated times and analysed for protein expression by immunoblotting. DMSO was used as a vehicle control and $\alpha$-tubulin as a loading control.

DMSO-treated cells, and between BMII siRNA- and control siRNA-transfected cells. Both BMI1 inhibitors and BMI1specific siRNA tended to reduce the expression of HES1 and MYC. DTX1 gene encoding Deltex E3 ubiquitin ligase 1 which antagonizes NOTCH signaling (18) tended to be also down-regulated in Jurkat cells. The limitation of these results is that changes over a longer period of time are not known as the analysis was performed only in cells treated for $24 \mathrm{~h}$ where there was a relatively small change in gene expression. 
Table I. Summarized results of expression of BMI1 and NOTCH signaling proteins in leukemia cells treated with BMI1 inhibitors shown in Figure 3.

\begin{tabular}{|c|c|c|c|c|c|c|c|}
\hline & & Jurkat & KOPT-K1 & THP-1 & TMD7 & OCI/AML2 & OCI/AML5 \\
\hline \multirow[t]{4}{*}{ ART } & BMI1 & $\downarrow$ & $\rightarrow$ & $\rightarrow$ & $\rightarrow$ & $\rightarrow$ & $\rightarrow$ \\
\hline & NOTCH1 & $\downarrow$ & $\rightarrow$ & $\rightarrow$ & $\rightarrow$ & $\rightarrow$ & $\rightarrow$ \\
\hline & MYC/HES1 & $\downarrow / \downarrow$ & $\rightarrow / \rightarrow$ & $\rightarrow / \rightarrow$ & $\rightarrow / \rightarrow$ & $\rightarrow /-$ & $\rightarrow /-$ \\
\hline & Cleaved-Casp3 & - & - & - & - & - & - \\
\hline \multirow[t]{4}{*}{ PRT } & BMI1 & $\rightarrow$ & $\rightarrow$ & $\rightarrow$ & $\rightarrow$ & $\rightarrow$ & $\rightarrow$ \\
\hline & NOTCH1 & $\rightarrow$ & $\downarrow$ & $\rightarrow$ & $\rightarrow$ & $\downarrow$ & $\downarrow$ \\
\hline & MYC/HES1 & $\downarrow / \rightarrow$ & $\downarrow / \downarrow$ & $\rightarrow / \downarrow$ & $\downarrow /-$ & $\downarrow /-$ & $\downarrow /-$ \\
\hline & Cleaved-Casp3 & - & $\uparrow$ & $\uparrow$ & $\uparrow$ & $\uparrow$ & $\uparrow$ \\
\hline \multirow[t]{4}{*}{ PTC } & BMI1 & $\downarrow$ & $\downarrow$ & $\downarrow$ & $\downarrow$ & $\downarrow$ & $\downarrow$ \\
\hline & NOTCH1 & $\downarrow$ & $\downarrow$ & $\downarrow$ & $\downarrow$ & $\downarrow$ & $\downarrow$ \\
\hline & MYC/HES1 & $\downarrow / \downarrow$ & $\downarrow / \downarrow$ & $\downarrow / \downarrow$ & $\downarrow / \downarrow$ & $\downarrow /-$ & $\downarrow /-$ \\
\hline & Cleaved-Casp3 & - & - & - & $\uparrow$ & - & $\uparrow$ \\
\hline
\end{tabular}

Upwards arrows, downwards arrows, rightwards arrows, and minus signs indicate up-regulation, down-regulation, no significant change, and no expression, respectively.

\section{Discussion}

We showed that BMI1 is expressed in leukemia cell lines and that BMI1 inhibitors slowed cell proliferation through induction of apoptosis, suggesting that BMI1 is involved in leukemia cell growth. It is well known that the major target of BMI1 is the CDKN2A gene encoding $\mathrm{p} 16^{\mathrm{INK} 4 \mathrm{~A}}$ and $\mathrm{p} 14^{\mathrm{ARF}}$ (5). However, in five of the examined cell lines, the $C D K N 2 A$ gene was deleted, suggesting that BMI1 inhibitors exerted their effects not through the restoration of $\mathrm{p} 16^{\mathrm{INK} 4 \mathrm{~A}}$ and $\mathrm{p} 14^{\mathrm{ARF}}$ expression. This notion was also supported by the finding that in $\mathrm{p} 16^{\mathrm{INK} 4 \mathrm{~A}}$-expressing TMD7 cells, the inhibitors did not up-regulate $\mathrm{p} 16^{\mathrm{INK} 4 \mathrm{~A}}$.

Recently, it was reported that several molecules such as cyclin G2 are targeted by BMI1 independently from $\mathrm{p} 16^{\mathrm{INK} 4 \mathrm{~A}}$ and $\mathrm{p} 14^{\mathrm{ARF}}(15)$. In this study, we examined NOTCH signaling known to affect BMI1 (16). We found that BMI1 inhibitors reduced protein expression of NOTCH-regulated proteins such as NOTCH1, cleaved NOTCH1, HES1, and MYC not only in T-ALL cells but also in AML cells. Our results indicated that this downregulation is not due to the off-target effects of BMI1 inhibitors, because BMII knockdown by siRNA also suppressed NOTCH1 signaling. These findings suggest that the NOTCH pathway acts downstream of BMI1 (Figure 5).

These findings are in contrast to previous reports on the interaction between BMI1 and $\mathrm{NOTCH}$, showing that NOTCH activation up-regulates BMI1 expression in T cells and intestinal stem cells, i.e., that NOTCH acts upstream of BMI1 $(16,17)$. To the best of our knowledge, this is the first study to show that BMI1 regulates NOTCH signaling. Given that, the NOTCH pathway is crucial for the growth of T-ALL cells (8), our findings suggest that NOTCH down-regulation

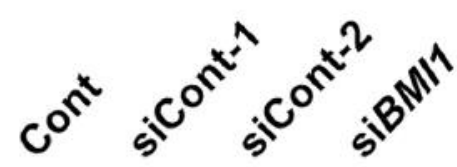

BMI1

NOTCH1

Cleaved

NOTCH1

MYC

\section{$\alpha-T u b u l i n$}

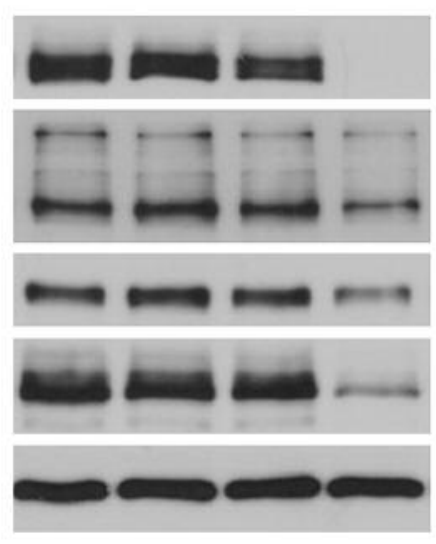

\section{p16 \\ HES1 \\ Caspase-3 \\ Cleaved \\ Caspase-3 \\ a-Tubulin}

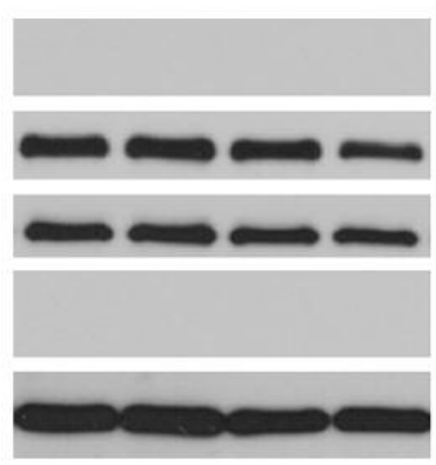

Figure 4. Effects of BMI1 knockdown by siRNA on the expression of BMII and NOTCH-related proteins. Jurkat cells were transfected with BMI1 siRNA (siBMI1) and two types of control siRNA (siCont) and analysed for the expression of the indicated proteins after $48 \mathrm{~h}$. 
Table II. Effects of BMI1 inhibitors and BMII siRNA on the expression of representative NOTCH-related genes examined by microarrays.

\begin{tabular}{lrrrrrrr}
\hline \multirow{2}{*}{ Genes } & \multicolumn{3}{c}{ Jurkat } & & \multicolumn{3}{c}{ THP-1 } \\
\cline { 2 - 4 } \cline { 6 - 8 } & ART & PTC & siRNA & PRT & PTC & siRNA \\
\hline NOTCH1 & -0.19 & -0.57 & -0.26 & -0.39 & -0.41 & 0.01 \\
HES1 & -0.48 & -2.41 & -1.01 & & -1.22 & -0.54 & -0.16 \\
MYC & 0.29 & -2.74 & -0.65 & & -1.12 & -1.07 & -0.58 \\
DTX1 & -0.41 & -1.19 & -0.73 & & N.S. & N.S. & N.S. \\
\hline
\end{tabular}

Numbers indicate $\log _{2}$ ratios of mRNA expression in leukemia cells treated with artemisinin (ART), PTC-209 (PTC), PRT4165 (PRT), or $B M I 1$ siRNA (siRNA) for $24 \mathrm{~h}$ normalized to that in DMSO-treated or control siRNA-treated cells. N.S.: Not significantly expressed.

by BMI1 inhibitors may be used to suppress proliferation of T-ALL cells. However, it is unclear whether BMI1 inhibitors slowed proliferation of leukemic cells through apoptosis related to the down-regulation of NOTCH. In terms of mechanisms of apoptosis, PRT4165 induced caspase-3 cleavage in five cell lines, whereas PTC-209 induced it only in two. More studies should be performed to further understand the molecular mechanisms linking BMI1 inhibition, NOTCH signaling, and growth of leukemic cells.

Considering that BMI inhibitors did not significantly affect the viability of normal lymphocytes, our data indicate that they could be candidates for novel molecular targeting drugs against leukemia. Because BMI1 regulates cell stemness $(5,6)$, BMI inhibitors could be used as drugs targeting leukemia stem cells. However, more research should be conducted to clarify molecular pathways targeted by BMI inhibitors and ensure their safety for normal hematopoietic stem cells.

\section{Acknowledgements}

This study was supported in part by a Grant-in-Aid for Scientific Research (C) from the Japan Society for the Promotion of Science (No. 26460669).

\section{References}

1 Cao R, Wang L, Wang H, Xia L, Erdjument-Bromage H, Tempst P, Jones RS and Zhang Y: Role of histone H3 lysine 27 methylation in Polycomb-group silencing. Science 298: 10391043, 2002.

2 Wang H, Wang L, Erdjument-Bromage H, Vidal M, Tempst P, Jones RS and Zhang Y: Role of histone H2A ubiquitination in Polycomb silencing. Nature 431: 873-878, 2004.

3 Cao L, Bombard J, Cintron K, Sheedy J, Weetall ML and Davis TW: BMI1 as a novel target for drug discovery in cancer. J Cell Biochem 112: 2729-2741, 2011.

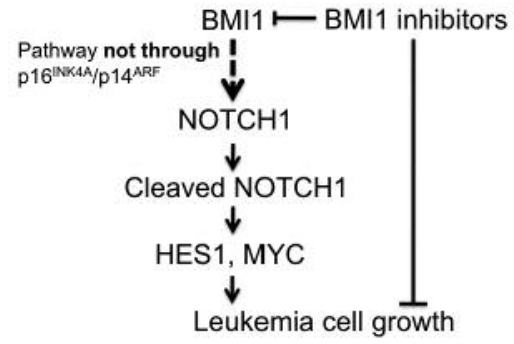

Figure 5. Schematic representation of the results obtained in this study.

4 Yuan J, Takeuchi M, Negishi M, Oguro H, Ichikawa $\mathrm{H}$ and Iwama A: Bmil is essential for leukemic reprogramming of myeloid progenitor cells. Leukemia 25: 1335-1343, 2011.

5 Rizo A, Olthof S, Han L, Vellenga E, de Haan G and Schuringa JJ: Repression of BMI1 in normal and leukemic human CD34(+) cells impairs self-renewal and induces apoptosis. Blood 114: 1498-1505, 2009.

6 Nishida Y, Maeda A, Chachad D, Ishizawa J, Qiu YH, Kornblau SM, Kimura S, Andreeff M and Kojima K: Preclinical activity of the novel B-cell-specific Moloney murine leukemia virus integration site 1 inhibitor PTC-209 in acute myeloid leukemia: Implications for leukemia therapy. Cancer Sci 106: 1705-1713, 2015.

7 Kreso A, van Galen P and Pedley NM: Self-renewal as a therapeutic target in human colorectal cancer. Nat Med 20: 2936, 2014.

8 Bolomsky A, Schlangen K, Schreiner W, Zojer N and Ludwig H: Targeting of BMI-1 with PTC-209 shows potent antimyeloma activity and impairs the tumour microenvironment. J Hematol Oncol 9: 17, 2016.

9 Tohda S: NOTCH signaling roles in acute myeloid leukemia cell growth and interaction with other stemness-related signals. Anticancer Res 34: 6259-6264, 2014.

10 Tohda S, Sakano S, Ohsawa M, Murakami N and Nara N: A novel cell line derived from de novo acute myeloblastic leukaemia with trilineage myelodysplasia which proliferates in response to a NOTCH ligand, Delta-1 protein. Br J Haematol 117: 373-378, 2002.

11 Tohda S, Curtis JE, McCulloch EA and Minden MD: Comparison of the effects of all-trans and cis-retinoic acid on the blast stem cells of acute myeloblastic leukemia in culture. Leukemia 6: 656-661, 1992.

$12 \mathrm{Wu}$ J, Hu D and Yang G: Down-regulation of BMI-1 cooperates with artemisinin on growth inhibition of nasopharyngeal carcinoma cells. J Cell Biochem 112: 1938-1948, 2011.

13 Alchanati I, Teicher C, Cohen G, Shemesh V, Barr HM, Nakache P, Ben-Avraham D, Idelevich A, Angel I, Livnah N, Tuvia S, Reiss Y, Taglicht D and Erez O: The E3 ubiquitin-ligase Bmi1/ Ring1A controls the proteasomal degradation of Top2alpha cleavage complex-a potentially new drug target. PLoS One 4: e8104, 2009.

14 Ismail IH, McDonald D, Strickfaden H, Xu Z and Hendzel MJ: A small molecule inhibitor of polycomb repressive complex 1 inhibits ubiquitin signaling at DNA double-strand breaks. J Biol Chem 288: 26944-26954, 2013. 
15 Mourgues L, Imbert V, Nebout M, Colosetti P, Neffati Z, Lagadec P, Verhoeyen E, Peng C, Duprez E, Legros L, Rochet N, Maguer-Satta V, Nicolini FE, Mary D and Peyron JF: The BMI1 polycomb protein represses cyclin G2-induced autophagy to support proliferation in chronic myeloid leukemia cells. Leukemia 10: 1993-2002, 2015.

16 López-Arribillaga E, Rodilla V, Pellegrinet L, Guiu J, Iglesias M, Roman AC, Gutarra S, González S, Muñoz-Cánoves P, Fernández-Salguero P, Radtke F, Bigas A and Espinosa L: Bmil regulates murine intestinal stem cell proliferation and selfrenewal downstream of Notch. Development 142: 41-50, 2015.

17 Schaller MA, Logue H, Mukherjee S, Lindell DM, Coelho AL, Lincoln P, Carson WF 4th, Ito T, Cavassani KA, Chensue SW,
Hogaboam CM, Lukacs NW and Kunkel SL: Delta-like 4 differentially regulates murine CD4 T cell expansion via BMI1. PLoS One 5: e12172, 2010.

18 Zhang P, Yang Y, Nolo R, Zweidler-McKay PA and Hughes DP: Regulation of NOTCH signaling by reciprocal inhibition of HES1 and Deltex 1 and its role in osteosarcoma invasiveness. Oncogene 29: 2916-2926, 2010.

Received May 9, 2017

Revised May 22, 2017

Accepted May 23, 2017 